\title{
Characterization of Tapeworm Metabolites and Their Reported Biological Activities
}

\author{
Phurpa Wangchuk ${ }^{1, *}$, Constantin Constantinoiu ${ }^{2}$, Ramon M. Eichenberger ${ }^{3}{ }^{(}$, Matt Field ${ }^{1,4}(\mathbb{C}$ \\ and Alex Loukas ${ }^{1}$ \\ 1 Centre for Molecular Therapeutics, Australian Institute of Tropical Health and Medicine, \\ James Cook University, Cairns, QLD 4878, Australia; matt.field@jcu.edu.au (M.F.); \\ alex.loukas@jcu.edu.au (A.L.) \\ 2 College of Public Health, Medical \& Vet Sciences, James Cook University, Townsville, QLD 4811, Australia; \\ constantin.constantinoiu@jcu.edu.au \\ 3 Institute of Parasitology, Vetsuisse faculty, University of Zurich, Winterthurerstrasse 266a, CH-8057 Zurich, \\ Switzerland; ramon.eichenberger@uzh.ch \\ 4 John Curtin School of Medical Research, Austalian National University, Canberra, ACT 2600, Australia \\ * Correspondence: phurpa.wangchuk@jcu.edu.au; Tel.: +61-7-42321249
}

Received: 21 March 2019; Accepted: 12 April 2019; Published: 15 April 2019

\begin{abstract}
Parasitic helminths infect billions of people, livestock, and companion animals worldwide. Recently, they have been explored as a novel therapeutic modality to treat autoimmune diseases due to their potent immunoregulatory properties. While feeding in the gut/organs/tissues, the parasitic helminths actively release excretory-secretory products (ESP) to modify their environment and promote their survival. The ESP proteins of helminths have been widely studied. However, there are only limited studies characterizing the non-protein small molecule (SM) components of helminth ESP. In this study, using GC-MS and LC-MS, we have investigated the SM ESP of tapeworm Dipylidium caninum (isolated from dogs) which accidentally infects humans via ingestion of infected cat and dog fleas that harbor the larval stage of the parasite. From this D. caninum ESP, we have identified a total of $49 \mathrm{SM}$ ( 35 polar metabolites and 14 fatty acids) belonging to 12 different chemotaxonomic groups including amino acids, amino sugars, amino acid lactams, organic acids, sugars, sugar alcohols, sugar phosphates, glycerophosphates, phosphate esters, disaccharides, fatty acids, and fatty acid derivatives. Succinic acid was the major small molecule present in the D. caninum ESP. Based on the literature and databases searches, we found that of 49 metabolites identified, only 12 possessed known bioactivities.
\end{abstract}

Keywords: Helminths; tapeworm; excretory-secretory products; small molecules; bioactivities

\section{Introduction}

Tapeworms are platyhelminth parasites that cause devastating diseases in both humans and livestock, such as neurocysticercosis and hydatid disease [1]. Human infections with tapeworms occur when people eat raw or undercooked infected beef and pork or by ingesting tapeworm eggs. Humans also occasionally get infected with the dog/cat flea tapeworm, Dipylidium caninum, by ingesting the cysticercoid stage which is found in dog or cat fleas (Ctenocephalides spp.) [2]. The method of transmission to humans is usually a result of close contact between children and their infected pets. In the small intestine of the vertebrate host the cysticercoid develops into the adult tapeworm which reaches maturity about one month after infection. The adult tapeworms measure up to $60 \mathrm{~cm}$ in length and resides in the small intestine where they attach to the gut wall using their scolex. The adult tapeworm releases proglottids (or segments) from its terminal end as they become gravid. 
The proglottids then migrate to the anus and are passed in the stool. Most D. caninum infections of dogs, cats and humans are asymptomatic and the infection is readily treated with praziquantel [1,2].

While these parasites feed in the small bowel, the adult tapeworms actively release excretory-secretory products (ESP) into the mucosa to modify their environment and circumvent the immune response of the host [3]. Tapeworm ESP have been shown to suppress inflammation by inducing regulatory B cells [4], tolerogenic dendritic cells [5], and regulatory T cells [6] which produce suppressor cytokines that keep inflammatory $\mathrm{T}$ cells and their effector molecules under control. These immunoregulatory strategies protect gastrointestinal tapeworms (such as D. caninum) from being ejected by the immune response but also provide protection to the host (only when present in moderate numbers) against gastrointestinal inflammatory diseases that result from a dysfunctional immune system. For example, the high molecular weight extracts of the rat tapeworm, Hymenolepis diminuta, has been shown to protect mice against experimental colitis and arthritis $[7,8]$.

Helminth ESP comprise mainly proteins, nucleic acids, glycans, lipids, extracellular vesicles and small molecules (SMs), which are released in a variety of "packages" to achieve a range of biological functions. To date, most attention has been afforded to characterising the protein components of ESP from helminths, and we know remarkably little about their non-protein SM components [9,10]. SM are responsible for many essential biological functions. Where investigated, lipids and glycans produced by helminths are restricted to different developmental phases of the parasite's lifecycle, implying tailored expression profiles for distinct functions in different environmental niches. For example, in the human gastrointestinal nematode Ascaris lumbricoides, ecdysones (steroidal prohormone) facilitate molting, and dimethylheptatriacontanes are involved in sexual communication [11]. Understanding the overall profile of helminth SM metabolites, which can be characterized using metabolomics techniques, will facilitate development of new tools for the diagnosis and treatment of helminth infections, as well as the development of novel therapeutics for treating inflammatory diseases [12]. Understanding metabolite expression patterns and identifying the SM components of ESP could provide important information on the molecular basis of parasitism and shed light on the various physiological processes that govern host-parasite interactions [9].

We recently characterised the secreted and somatic SM complement of gastrointestinal hookworms and showed that these worms secrete SM that suppress inflammation in a mouse model of inflammatory bowel diseases and reduce inflammatory cytokine secretion by human peripheral blood mononuclear cells [13]. In this study herein, we have used gas chromatography mass spectrometry (GC-MS) and liquid chromatography mass spectrometry (LC-MS) to characterize the ESP metabolome of D. caninum and searched the literature and public databases for reported biological functions/roles/properties of the identified SM, with a particular focus on anti-inflammatory properties.

\section{Results}

\subsection{Polar Metabolites of D. caninum ESP Identified Using GC-MS}

Adult D. caninum were collected from dogs and were cultured in a single component glutamax culture medium with antimycotic/antibiotic. Glutamax is a simple medium that consists of $200 \mathrm{mM}$ L-alanyl-L-glutamine dipeptide in saline. Culture medium containing ESP was filtered $(<10 \mathrm{kDa}$ centrifugal filter) to obtain small molecule extracts, lyophilized, fractionated and then analysed using GC-MS and LC-MS. While GC-MS was used for identifying targeted polar and fatty acid components, LC-MS was used for analysing the targeted short chain fatty acids (SCFA). The ESP of D. caninum was fractionated to obtain a biphasic partition of the solution: upper aqueous phase (methanol:water) and lower organic phase (chloroform), which were derivatized and analyzed for polar and non-polar metabolites using GC-MS and LC-MS.

For GC-MS analysis, an Agilent's Mass Hunter Quantitative Analysis software (v.7), Mass Hunter Library (MHL), in-house Metabolomics Australia metabolite library (MAML), NIST library and the Metabolomics Standard Initiative (MSI) level 1 was used for identification and validation of compounds. 
Each EI-MS spectra $\left[\mathrm{M}^{+}\right]$of a metabolite was matched with EI-MS spectra in the compound library. The MAML was developed by running authentic standards in the GC-MS prior to running test samples. This same method was used for running our samples in the GC-MS. ${ }^{13} \mathrm{C}$-sorbitol and ${ }^{13} \mathrm{C}^{15} \mathrm{~N}$-valine were used as internal standards, which helped in determining the relative concentrations of each metabolite by comparing the metabolite peak areas with the peak areas of the internal standards. Using these techniques, we identified 35 metabolites from the polar fraction (Table 1) of D. caninum ESP. The top 10 small polar metabolites (identified based on their concentration/peak areas) include succinic acid (major component) followed by lactic acid $>$ myo-inositol $>$ scyllo-inositol $>$ tyrosine $>$ malic acid $>$ talose $>$ glucose $>$ glycerol $>$ phenylalanine. Galactose-6-phosphate was the least abundant metabolite present in the polar ESP fraction.

Table 1. Total polar small molecule (SM) identified by gas chromatography mass spectrometry (GC-MS) from the excretory-secretory products (ESP) of D. caninum with their molecular characteristics.

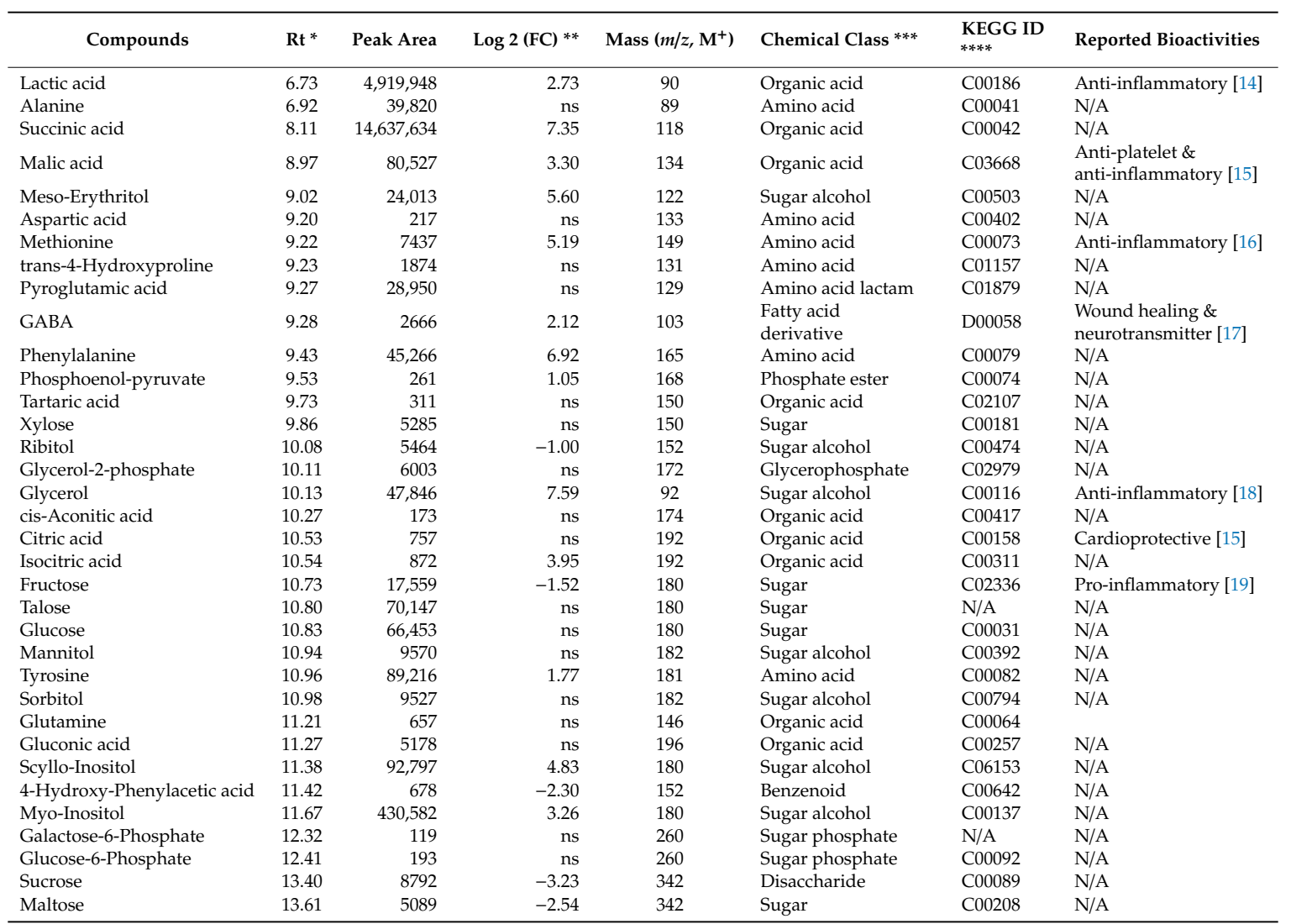

${ }^{*} \mathrm{Rt}=$ retention time in minute. ${ }^{* *} \log 2$ of volcano plot (Metaboanalyst 4 ) represent the mean ratio fold change plotted on a $\log 2$ scale (log transformed) of the relative abundance of each metabolite between two samples, so that same fold change (up/down regulated or $+/-$ ) will have the same distance to the zero baseline. The values were taken from and shows the significant metabolites present in the ESP. ${ }^{* * *}$ chemical class was taken from HMDB (http://www.hmdb.ca/). ${ }^{* * * *}$ KEGG ID (https://www.genome.jp/kegg/) provides the information on the biosynthetic and metabolic pathways of a compound. ID = identity; ns = not significant; FC = fold change; N/A = not available.

Table 1 shows the retention times, peak areas, assigned individual masses, chemotaxonomy/ chemical groups and Kyoto Encyclopedia of Genes and Genomes (KEGG) identities (ID), as well as the reported biological properties of each SM. The KEGG ID offers information on SM metabolism and biosynthesis pathways. These SM metabolites belong to 11 different classes including amino acids, amino sugars, amino acid lactams, organic acids, sugars, sugar alcohols, sugar phosphates, glycerophosphates, phosphate esters, disaccharides, and fatty acid derivatives. The highest number of compounds identified belong to the chemical class of organic acids (nine molecules), followed by amino acids (6 molecules), sugar alcohol (seven molecules), sugar (four molecules), sugar phosphate 
(two molecules), and other minor chemical groups. Interestingly, the major SM present (as putatively determined from the chromatographable peak areas) in the D. caninum ESP was succinic acid.

\section{Statistical Analysis Using MetaboAnalyst 4.0 Software}

We performed a statistical data analysis (univariate and multivariate) on the polar metabolites identified in Table 1. First, a univariate volcano plot analysis was performed to compare the datasets of the two groups (D. caninum versus culture media) and understand which metabolites are significantly different between the samples. The volcano plot also calculated fold change and $p$-values that enabled comparison of absolute value changes between the two-group mean (result plotted in log 2 scale to obtain same distance to zero baseline). The variable is reported here as significant if the value is above a given count threshold ( $>75 \%$ of pairs/variable), and has a fold change of $>2$ and a $p$-value of $<0.05$. Table 1 shows the fold change and the $p$ values of these significantly different metabolites. For example, D. caninum ESP contains significant levels of succinic acid, meso-Erythritol, glycerol, and scyllo-Inositol

The volcano plot showed that 18 metabolites of the D. caninum ESP were significantly different in their concentration from culture media-derived metabolites (fold change $>2, p$ value $<0.05$ ) in their peak intensities (Figure 1).

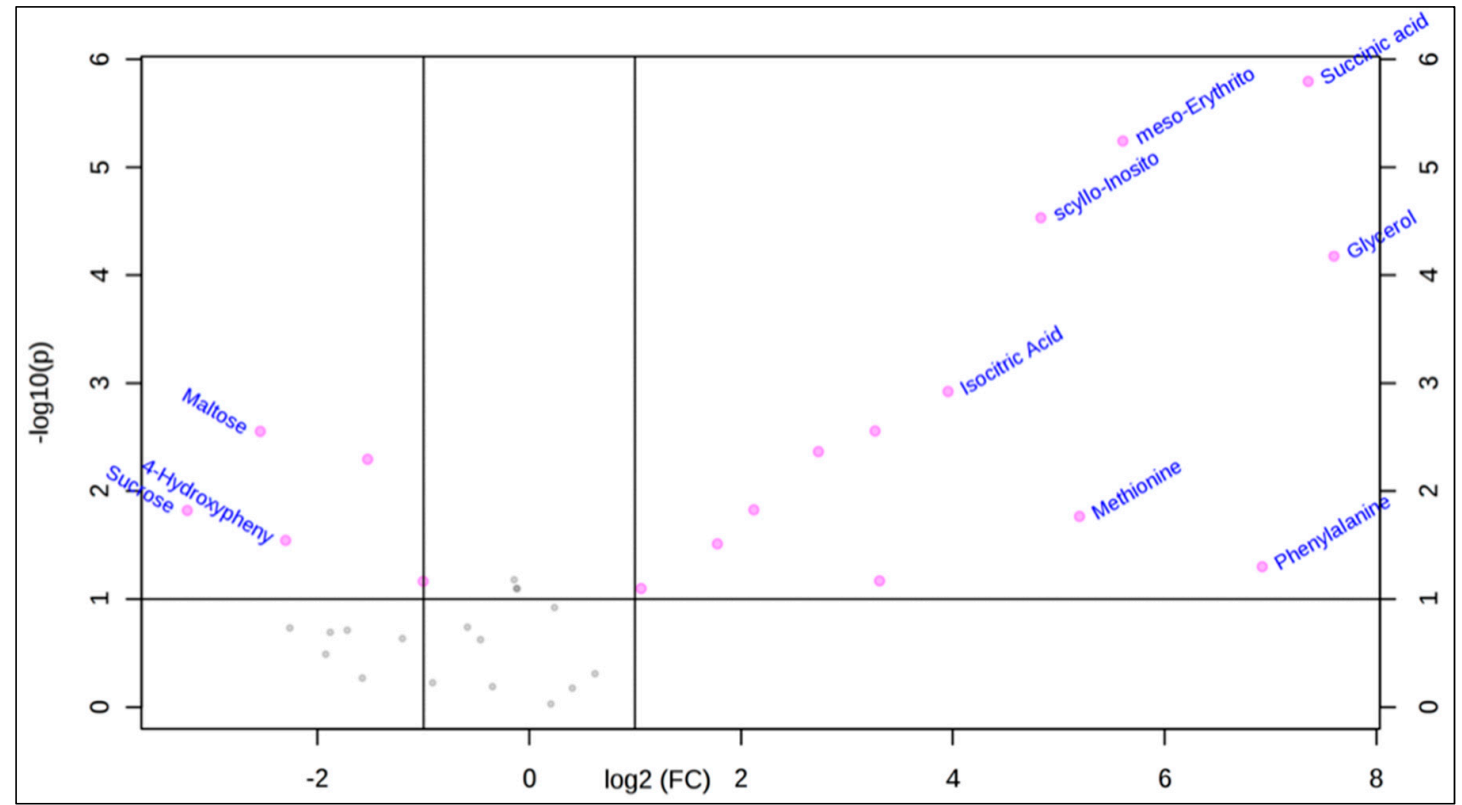

Figure 1. Univariate volcano plot analysis with fold change threshold (x) 2 and t-tests threshold (y) 0.1 showing metabolites with significantly different abundance between $D$. caninum ESP and culture medium. The further the metabolite position is from $(0,0)$, the more significant the difference in metabolite presence (pink dots). Data analyzed by MetaboAnalyst 4 (samples normalized by mean, $\log$ transformation and autoscaling).

In addition to univariate analysis, we performed multivariate chemometric analysis (applied to three sample groups) on the targeted matrix extracted, which included the identified metabolites. The PCA plot (Figure 2a) showed clear separation between the three sample groups of D. caninum ESP, glutamax medium control and pooled biological replicates of D. caninum ESP and culture media. Such clear separation of the groups indicated that helminth metabolites and culture media (glutamax) components could be readily distinguished and that D. caninum produces an array of metabolites when cultured in minimal medium. The partial least square discriminant analysis (PLS-DA) scores (Figure 2b) shows top 15 metabolites present in D. caninum ESP and medium. 

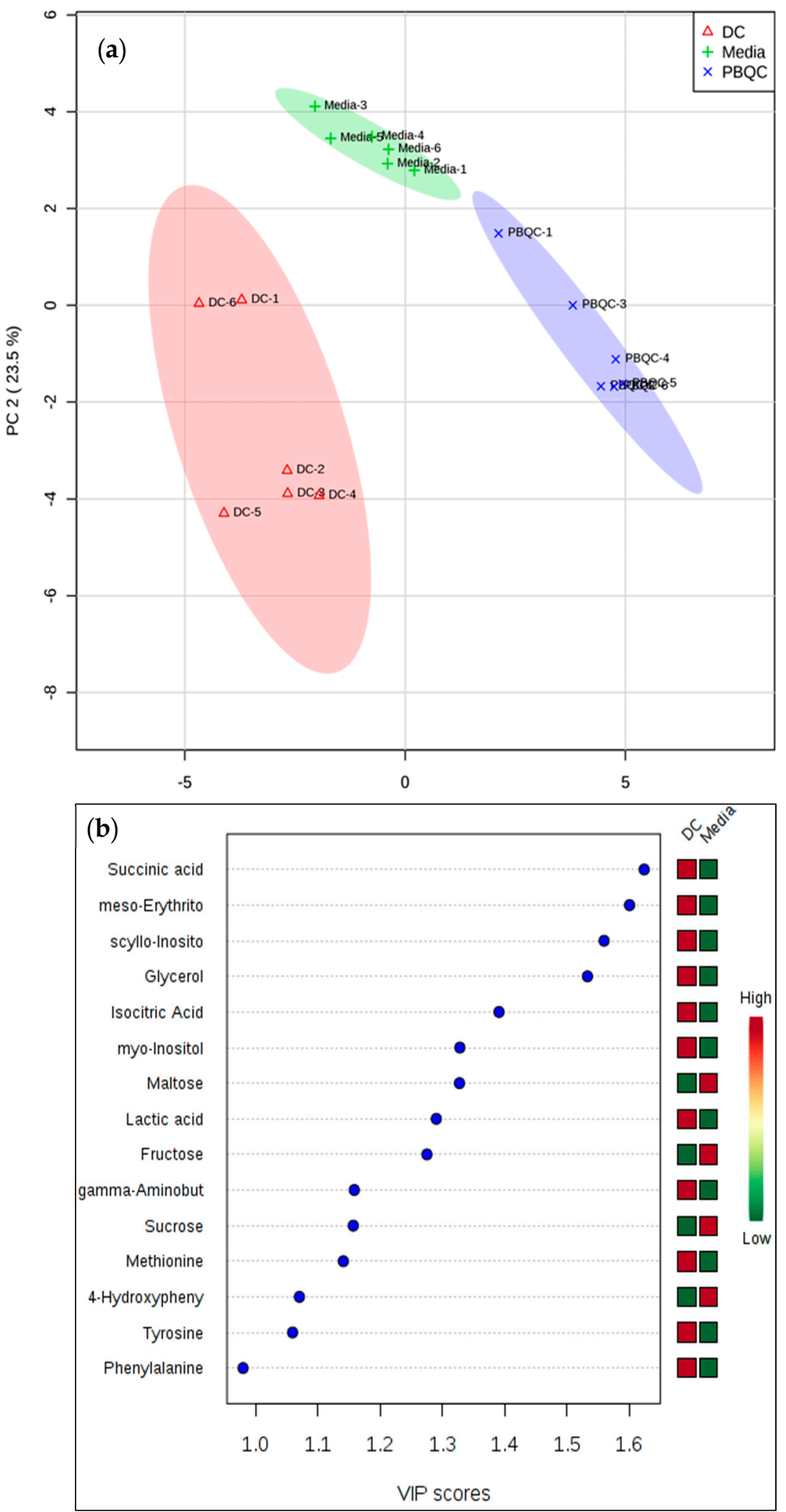

Figure 2. Multivariate principal component analysis (PCA) of D. caninum ESP. (a) PCA scores plot showing clear separation of three sample groups (D. caninum, media control and pooled biological replicates of ESP and medium samples). (b) Important features identified by PLS-DA. The colored boxes on the right indicate the relative peak intensities of the corresponding metabolite in D. caninum ESP and culture medium (15 important SM). Data analyzed by MetaboAnalyst 4 (samples normalized by mean, log transformation, and autoscaling). 
Hierarchical cluster analysis of the three sample groups produced a heat map of metabolites (Figure 3) that revealed the types of metabolites released into the media. The heat map shows distinct separation between the SM complements of the ESP compared to culture media. For example, while succinic acid, glycerol, and meso-erythritol were abundantly present in the ESP of D. caninum, these metabolites were not presented in the culture medium. On the other hand, fructose, ribitol, and maltose were presented in higher concentrations/intensities in culture medium, but were absent in D. caninum ESP.

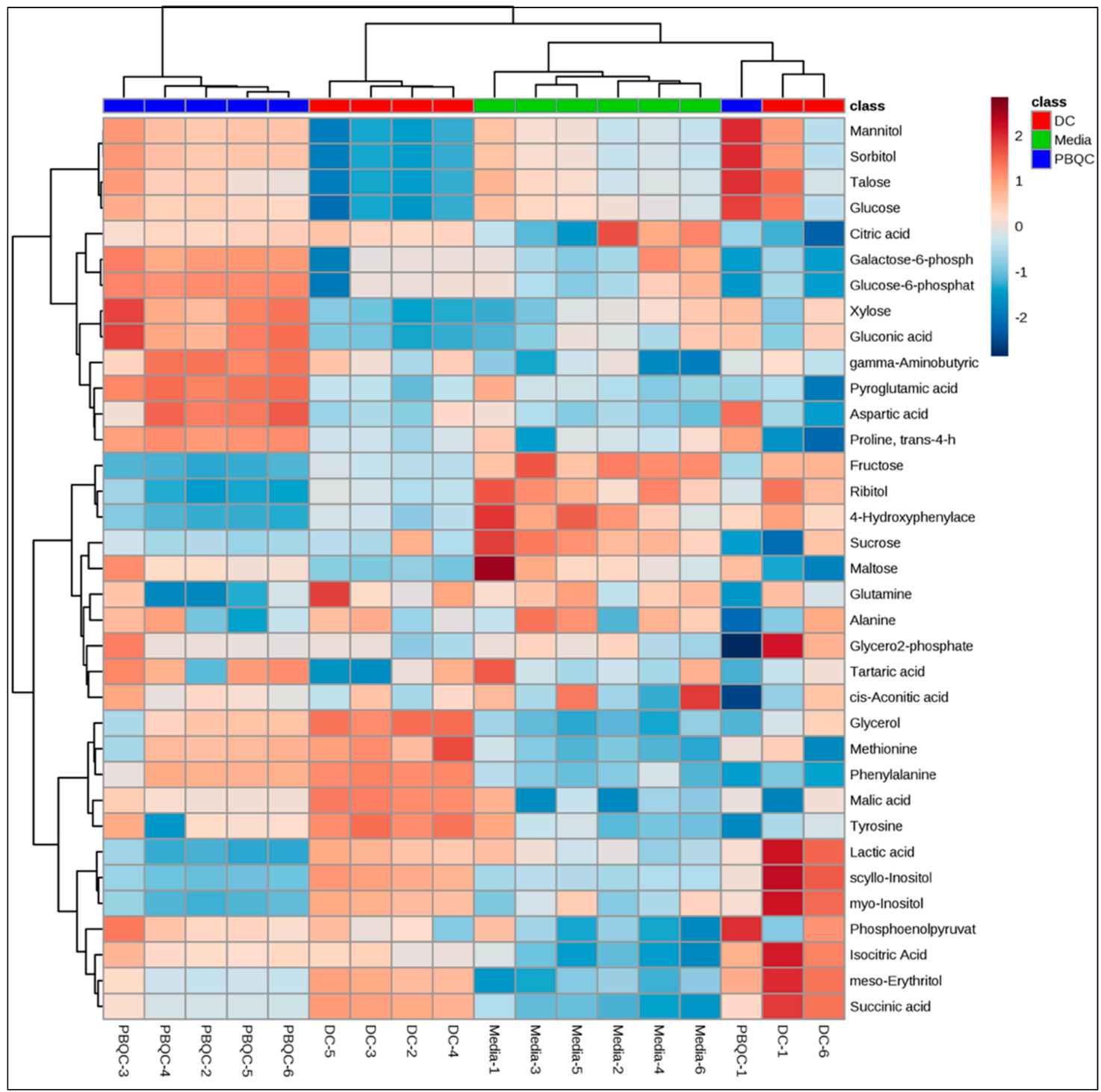

Figure 3. Heat map showing polar metabolites of D. caninum ESP. Heat map was generated using MetaboAnalyst 4 ( $N=6$ replicates). Deep brown denotes highest peak areas of metabolites and deep blue denotes lowest peak areas or complete absence of metabolites.

\subsection{Non-Polar Metabolites of D. Caninum Identified Using GC-MS and LC-MS}

From the non-polar chloroform fractions of D. caninum, we identified 14 fatty acids ranging in lipidic carbon numbers $\mathrm{C} 2-\mathrm{C} 22$, including saturated, un-saturated and short chain fatty acids (SCFA) (Table 2). ${ }^{13}$ C18:0 was used as an internal standard and the reference standards (C10-C30) were used for validation. Fatty acids were identified by library matching techniques as described in the polar metabolites section above. Stearic acid (C18:0) is the major fatty acid present in the ESP. Tridecylic acid (C13:0) is the second major component of the non-polar fraction of D. caninum ESP. 
Three polyunsaturated fatty acids (oleic acid, linoleic acid, and arachidonic acid) and only one SCFA (acetate (C2:0)) were detected in the D. caninum SM ESP.

Table 2. Fatty acids identified from the ESP of D. caninum with their lipid number, mass, KEGG identities, and reported biological properties.

\begin{tabular}{|c|c|c|c|c|c|}
\hline Fatty Acid Names. (Lipid Number) & $\mathbf{R t}$ * & Peak Areas & $\begin{array}{c}\text { Mass } \\
\left(m / z, \mathbf{M}^{+}\right)\end{array}$ & $\begin{array}{l}\text { KEGG } \\
\text { ID ** }\end{array}$ & $\begin{array}{l}\text { Reported Biological } \\
\text { Properties }\end{array}$ \\
\hline Undecanoic acid (C11:0) & 7.35 & 5503 & 186 & C17715 & $\mathrm{N} / \mathrm{A}$ \\
\hline Decanoic acid (C10:0) & 7.67 & 5507 & 172 & C01571 & N/A \\
\hline Tridecylic acid (C13:0) & 9.03 & 21,853 & 214 & N/A & $\mathrm{N} / \mathrm{A}$ \\
\hline Dodecanoic acid (C12:0) & 9.22 & 767 & 200 & C02679 & $\begin{array}{c}\text { Anti-inflammatory \& } \\
\text { antibacterial [20] }\end{array}$ \\
\hline Tetradecanoic acid (C14:0) & 11.29 & 1864 & 228 & C06424 & $\mathrm{N} / \mathrm{A}$ \\
\hline Pentadecylic acid (C15:0) & 12.53 & 3431 & 242 & C16537 & $\mathrm{N} / \mathrm{A}$ \\
\hline Hexadecanoic acid (C16:0) & 13.84 & 16,171 & 256 & C00249 & Anti-inflammatory [21] \\
\hline Heptadecanoic acid (C17:0) & 15.25 & 1238 & 270 & N/A & $\mathrm{N} / \mathrm{A}$ \\
\hline Octadecanoic acid (C18:0) & 15.25 & 32,883 & 284 & C01530 & Anti-inflammatory [22] \\
\hline Oleic acid (C18:1, 9Z- cis) & 17.16 & 1219 & 282 & C00712 & N/A \\
\hline Arachidonic acid (C20:4) & 18.15 & 2076 & 304 & C00219 & N/A \\
\hline Linoleic acid (C18:2) & 18.33 & 5336 & 280 & C01595 & $\mathrm{N} / \mathrm{A}$ \\
\hline Eicosanoic acid (C20:0) & 19.60 & 5981 & 312 & C06425 & N/A \\
\hline Docosahexaenoic acid (C22:6) & 26.84 & 6960 & 328 & C06429 & Anti-inflammatory [23] \\
\hline
\end{tabular}

* Rt $=$ retention time in minute. ${ }^{* *}$ KEGG ID (https://www.genome.jp/kegg/) provides the information on the biosynthetic and metabolic pathways of a compound. ID = identity; ns = not significant; FC = fold change; N/A = not available.

\subsection{Literature Review Reveals Bioactive Small Molecules Present in the D. caninum ESP}

Based on the literature and databases searches, we found that of 49 total metabolites identified (includes polar and lipid compounds), 12 of them (see Table 2) including glycerol [18], lactic acid [14], malic acid [15], methionine [16], octadecanoic acid [22], hexadacanoic acid [21], docosahexaenoic acid [23], acetate [24], and dodecanoic acid [20] were reported to have anti-inflammatory properties. Another four SM including citric acid [15], malic acid [15], aminobutyric acid [17], and fructose [19] were known to possess cardioprotective, anti-platelet aggregation, wound healing and pro-inflammatory activities, respectively. Dodecanoic acid [20] was also able to inhibit the growth of Staphylococcus aureus, Bacillus cereus, Salmonella typhimurium, and Escherichia coli.

\section{Discussion}

Gastrointestinal helminths affect humans, livestock and companion animals causing immense morbidity, growth retardation, and productivity losses, and yet very little is understood about the ESP of these helminths. ESP contains complex mixtures of compounds including macromolecules (inter alia proteins) and micromolecules (non-protein small molecules). Micromolecules or SM of the gastrointestinal helminths are largely unstudied and there is a paucity of information on metabolome composition of helminth ESP in general.

Recent advances in metabolomics technologies, which can identify and quantify cellular metabolites using sophisticated analytical tools including MS and nuclear magnetic resonance (NMR) spectroscopy, have ushered in a new era of data mining and interpretation. However, metabolomics applications to study helminths and their interactions with vertebrate hosts is still in its infancy [9], with most of the previous studies devoted to understanding the effect of parasitic infections on host metabolite expression [25]. Only limited studies have been reported $[9,26]$ on the metabolomes of blood flukes (Schistosoma mansoni) and gastrointestinal nematodes (Ascaris lumbricoides and Ancylostoma caninum), and until now there has been a paucity of information for tapeworms (cestodes). Other than fatty acids, none of the polar metabolites that were previously reported from S. mansoni and A. lumbricoides were detected here in D. caninum ESP. This variation in reported metabolite composition could be due to distinctiveness in the groups of helminths studied (spanning three different phyla), or differences in culture media, methods of ESP production, and metabolomics techniques used. 
It is known that in the free-living nematode Caenorhabditis elegans, the metabolism or expression of biochemicals is strongly dependent on diet and developmental stage [27].

Using a single component culture medium (glutamax), we have successfully cultured adult stage hookworms [13], whipworms, roundworms and now tapeworms ex vivo and collected their ESP. Recently, we reported 46 polar metabolites, 22 fatty acids and 5 SCFA from the somatic tissue extract of the dog hookworm A. caninum, and another 29 polar metabolites, 13 fatty acids and 6 SCFA from its ESP [13]. This current study on the ESP of $D$. caninum detected 35 polar metabolites with molecular weights $(\mathrm{m} / \mathrm{z}$ ) ranging from 89 to 342 atomic mass unit (amu), and 14 fatty acids with $\mathrm{m} / \mathrm{z}$ ranging from 60-328 amu. While we detected six SCFA from hookworm ESP [13], only acetate was detected in the ESP of D. caninum. Unlike nematodes (A. caninum), cestodes such as D. caninum do not have an advanced internal digestive system and instead adsorb nutrients across the tegument. Interestingly, while pyroglutamic acid was the major compound detected in A. caninum ESP [13], succinic acid was the major compound detected in D. caninum ESP. Variations in major metabolic pathways can be expected since the worms belong to completely unrelated phyla.

While the KEGG pathway analysis suggested that succinic acid is produced via tyrosine metabolism involving citrate cycle (TCA) pathways, pyroglutamic acid is produced via glutathione metabolism. Pyroglutamic acid is found in substantial amounts in brain, skin and plants [28]. Only one SCFA, acetate, was detected in the D. caninum ESP. The nature and origin of SCFA production/biosynthesis by helminths in general remains sketchy [13]. While it is possible that helminths synthesize SCFA de novo, the host microbiome could be a potential source of SCFA for tapeworms (assuming that antibiotic treatment did not completely kill all adhered bacteria upon removal from the canine host gut), since acetate, butyrate and propionate are known to be produced and utilized by bacteria [29]. Further studies will be needed to define the contribution of the commensal microbiome to SCFA synthesis and its detection in helminth ESP metabolites.

Helminths clearly have negative health impacts on many infected vertebrate hosts, but the recent spotlight on iatrogenic helminth therapy [30,31] and the discovery of immunoregulatory proteins [32] and more recently metabolites $[10,13]$ secreted by helminths has resulted in an appreciation of their pharmacopoeic properties. The ESP metabolites of $D$. caninum contain at least $12 \mathrm{SM}$ that possess known bioactivities with relevance to human health. Of particular interest, the unsaturated fatty acid, docosahexaenoic acid has demonstrated anti-inflammatory activities [23], the SCFA, acetate is important in regulating colonic blood flow and ileal motility [33] and other metabolic processes that govern inflammatory processes. Novel targeted approaches for delivering the three major SCFAs-acetate, propionate and butyrate - to the gut is of great interest for the treatment of inflammatory bowel diseases [34] and given the acceptance of iatrogenic helminth infection, it is tempting to speculate that human infection with $D$. caninum presents an opportunity for targeted gastrointestinal delivery of therapeutic SCFAs. We believe that these anti-inflammatory SM, individually or in combination, are an integral part of the multi-pronged immunoregulatory storm released by parasitic helminths, and hold promise as a novel platform of therapeutics inspired by host-parasite coevolution.

\section{Materials and Methods}

D. caninum (15-20 adult worms) was collected from stray dogs using methods we have described earlier for hookworms [13]. The worms were washed (5 times) with PBS containing $5 \%$ antibiotic/antimycotic (AA), transferred to pre-warmed culture media ( $2 \%$ glutamax in phosphate buffered saline, $2 \% \mathrm{AA}$ ) and were incubated for $6-7 \mathrm{~h}$ at $37^{\circ} \mathrm{C}$ in $5 \% \mathrm{CO}_{2}$. The ESP in culture media were collected, centrifuged at $1831 \times g$ for $20 \mathrm{~min}$ at $4{ }^{\circ} \mathrm{C}$ to remove cells/feces/debris, and the supernatant was filtered using $3 \mathrm{kDa}$ cut off Amicon ultra centrifugal filters (Merck Millipore, Ireland, $15 \mathrm{~mL}$ ) to obtain small molecule extracts. The samples were prepared for respective GC-MS and LC-MS analyses and were analyzed using the method described by us elsewhere [13]. 


\subsection{GC-MS Analysis of ESP Samples of D. caninum}

\subsubsection{Sample Preparation and Cryomill Extraction Methods}

The D. caninum ESP (20 mg) was placed in chilled cryomill tubes ( 5 biological replicates for each worm), suspended in $600 \mu \mathrm{L}$ of extraction solution of methanol:water $(3: 1, v / v)$ containing internal standard ${ }^{13} \mathrm{C}$-sorbitol (Sigma-Aldrich, Castle Hill, Australia), and then extracted using a Precellys 24 Cryolys unit (Bertin Technologies, avenue Ampère, France) at $6800 \mathrm{rpm}(3 \times 30 \mathrm{~s}$ pulses, $45 \mathrm{~s}$ interval between pulses) and $-10{ }^{\circ} \mathrm{C}$ temperature (pre-chilled with liquid nitrogen). The homogenate $(600 \mu \mathrm{L})$ was transferred to a clean microfuge tube (on ice) and $150 \mu \mathrm{L}$ pre-chilled chloroform was added to it. A monophasic mixture of chloroform:methanol:water $(1: 3: 1, v / v)$ was obtained by vortexing vigorously. The solution was chilled on ice for $10 \mathrm{~min}$ with regular mixing and then centrifuged for $5 \mathrm{~min}$ at $0{ }^{\circ} \mathrm{C}$. The supernatant was transferred to a fresh $1.5 \mathrm{~mL}$ microfuge tube on ice and milli-Q water $(300 \mu \mathrm{L})$ was added to each tube to obtain a biphasic partition of the solution (chloroform:methanol:water 1:3:3, $v / v$ ). The sample was vortexed vigorously and then centrifuged at $0{ }^{\circ} \mathrm{C}$ for a further 5 min. Both upper aqueous phase (methanol:water, $900 \mu \mathrm{L}$ ) and lower phase (chloroform) were derivatized and analyzed for polar and non-polar content respectively. A small amount of these samples were aliquoted for the pooled biological replicates (PBQC). The samples were grouped as 'D. caninum ESP', 'PBQC quality control' and 'media control'.

\subsubsection{Derivatization and Analysis of Polar Fraction Using Targeted GC-MS Technique}

From the upper aqueous phase, we aliquoted $50 \mu \mathrm{L}$ and transferred to a pulled point insert tubes and dried in an evaporator (Christ RVC 2-33 CD, John Morris Scientific, Chatswood, Australia) at $30{ }^{\circ} \mathrm{C}$. Another $50 \mu \mathrm{L}$ of the aqueous sample was added to the tubes every $30-40$ min until completely dry. Anhydrous methanol $(50 \mu \mathrm{L})$ was added to dehydrate the samples and then derivatized by adding $20 \mu \mathrm{L}$ methoxyamine (30 mg/mL in pyridine, Sigma Aldrich, Castle Hill, Australia) at $37^{\circ} \mathrm{C}$ for $30 \mathrm{~min}$, followed by addition of $20 \mu \mathrm{L} \mathrm{N}, \mathrm{O}$-Bis(trimethylsilyl) trifluoroacetamide (BSTFA) $+1 \%$ trimethyl-chlorosilane (TMCS) (ThermoFisher Scientific Australia) at $37^{\circ} \mathrm{C}$ for $30 \mathrm{~min}$. It was then left standing at room temperature for $2 \mathrm{~h}$ and $1 \mu \mathrm{L}$ of this derivatized polar sample was analyzed using an Agilent 7890 GC-MS (5973 MSD) [35]. Agilent VF-5 ms column (30 m $\times 0.25 \mathrm{~mm} \times 0.25 \mu \mathrm{m})$ was used for chromatographic separation of metabolites. The oven was set to $35^{\circ} \mathrm{C}$, held for $2 \mathrm{~min}$, then ramped at $25^{\circ} \mathrm{C} / \mathrm{min}$ to $325^{\circ} \mathrm{C}$ and then held for $5 \mathrm{~min}$. Helium was used as the carrier gas at a flow rate of $1 \mathrm{~mL} / \mathrm{min}$ and the GC-MS metabolite $\mathrm{m} / \mathrm{z}$ scanning range was set to 50-600 atomic mass unit (amu). Agilent's Mass Hunter Quantitative Analysis software (v.7) was used for analyzing targeted metabolomics data and the Metabolomics Standard Initiative (MSI) level 1 was used for confirmation. Each compound was identified by a library matching technique, in which an observed EI-MS spectra $\left[\mathrm{M}^{+}\right]$of each metabolite was matched with EI-MS spectra in the Mass Hunter Library (MHL) as well as in the NIST library. The in-house Metabolomics Australia metabolite library (MAML) was used to extract target ion peak areas (in a .csv data matrix) for polar metabolites. The MAML was developed by running authentic standards in the GC-MS prior to running test samples. This same method was used for running our samples in the GC-MS. ${ }^{13} \mathrm{C}$-sorbitol and ${ }^{13} \mathrm{C}^{15} \mathrm{~N}$-valine as were used as internal standards, which helped in determining the relative concentrations of each metabolite by comparing the metabolite peak areas with the peak areas of the internal standards.

\subsubsection{Analysis of Non-Polar Fraction Using Targeted GC-MS Approach}

The non-polar fraction/organic phase of each biological replicate sample was sealed into GC vials and trans-esterified to create fatty acid methyl esters. Gerstel MPS2 autosampler robot was used for mixing the samples with $5 \mu \mathrm{L}$ Methprep-II (Alltech). The solution was incubated for $30 \mathrm{~min}$ at $37^{\circ} \mathrm{C}$ with slow shaking. Agilent 7890 gas chromatograph coupled to a triple quadrupole mass selective detector (Agilent Technologies, Australia) was used for sample analyses. Samples (2 L) were injected in split mode (10:1) into an inlet set at $250{ }^{\circ} \mathrm{C}$. The chromatographic separation was achieved on a 
SGE BPX70 column $(60 \mathrm{~m} \times 0.25 \mathrm{~mm}$ i.d. $\times 0.25 \mathrm{um}$ film thickness) (Trajan Scientific and Medical, Ringwood, Australia). The oven conditions were initially set to $70^{\circ} \mathrm{C}$ for $1 \mathrm{~min}$, then gradually raised to $150{ }^{\circ} \mathrm{C}\left(40{ }^{\circ} \mathrm{C} / \mathrm{min}\right), 200{ }^{\circ} \mathrm{C}\left(4{ }^{\circ} \mathrm{C} / \mathrm{min}\right), 220{ }^{\circ} \mathrm{C}\left(3{ }^{\circ} \mathrm{C} / \mathrm{min}\right), 250{ }^{\circ} \mathrm{C}\left({ }^{\circ} \mathrm{C} / \mathrm{min}\right)$ and finally held at $250{ }^{\circ} \mathrm{C}$ for $4 \mathrm{~min}$. Helium was used as carrier gas (flow was constant at $1.5 \mathrm{~mL} / \mathrm{min}$ ) and the MS transfer line was set at $280^{\circ} \mathrm{C}$. Compounds were ionized using electron impact fragmentation $(-70 \mathrm{eV})$ and mass spectra were collected in scan mode over the $m / z$ range of 50-450 at 3.6 scans/s. Each compound ion was recorded in a positive mode $\left(\mathrm{M}^{+}\right)$and was identified by a library matching technique. The target ion peak areas were extracted using the in-house MHFAL and output as a data matrix in the required format (.csv) for further analysis. ${ }^{13} \mathrm{C} 18: 0$ was used as an internal standard.

\subsection{LC-MS Identification of SCFA}

We used the LC-MS protocols described previously [13,36]. The ESP samples were extracted using the 'Cryomill extraction method' and a small portion $(20 \mu \mathrm{L})$ of the extract was derivatized using 3-nitrophenylhydrazine (3-NPH), which converted SCFAs to their 3-nitrophenylhydrazones. The samples $(1 \mu \mathrm{L})$ were then injected into a Shimadzu LC 30AD-TQ 8060 triple quadrupole mass spectrometer and analysed in triplicates. Authentic SCFA standards (including acetate, propionate, isobutyrate, butyrate, 2-methylbutyrate, isovalerate, valerate, 3-methylvalerate, isocaproate, and caproate) were run alongside samples in multiple reaction monitoring mode in order to facilitate Metabolomics Standard Initiative (MSI) level 1 confirmation. An Agilent EC-C18 poroshell 120 (50 $\mathrm{mm} \times 2.1 \mathrm{~mm} \times 2.7 \mu \mathrm{m}$ column) was used for the analysis, using $100 \%$ water $(+0.1 \%$ formic acid) mobile phase A and $100 \%$ acetonitrile ( $+0.1 \%$ formic acid) mobile phase $\mathrm{B}$. The column flow rate was $0.55 \mathrm{~mL} / \mathrm{min}$, with the column temperature kept at $40{ }^{\circ} \mathrm{C}$. The elution gradient was optimized at $15 \% \mathrm{~B}$ for $1 \mathrm{~min}, 15-25 \%$ B in $7 \mathrm{~min}, 25-30 \%$ in $1 \mathrm{~min}, 30-100 \%$ in $3 \mathrm{~min}$, held at $100 \%$ for $3 \mathrm{~min}$, then back to starting conditions and held for $2 \mathrm{~min}$-providing a total run time of $17 \mathrm{~min}$. The MRM transitions were optimized by direct infusion of the derivatives from a mixed standard solution containing each fatty acid. Collision energies were optimized $(22 \mathrm{eV}, 25 \mathrm{eV}$, and $28 \mathrm{eV})$ for each analyte and the most sensitive energies were selected for the analysis. Each ion was recorded in positive mode $\left[\mathrm{M}^{+}\right]$and were identified as described above.

\subsection{Data Analyses and Statistical Interpretation}

The GC-MS data was analyzed in a targeted approach using Agilent's Mass Hunter Quantitative Analysis software (v.7). Target ion peak areas for polar and non-polar metabolites were extracted using the in-house Metabolomics Australia metabolite library and output as a data matrix (in .csv format) for further analysis. Chemometric and statistical analyses were undertaken using MetaboAnalyst 4 [37]. The GC-MS data was normalised with respect to median and log transformed prior to performing chemometric analyses including Principal Component Analyses (PCA), Partial Least Squares-Discriminant Analysis (PLS-DA) and cluster heatmap analyses (compound concentration versus samples in 5 replicates).

\subsection{Literature Searches and Their Content Analyses Focusing on Anti-Inflammatory Properties}

The small molecules identified from the D. caninum ESP were queried using: (1) Human metabolome database (HMDB), which contains 114,100 metabolite entries including both water-soluble and lipid soluble metabolites [28]; (2) DrugBank, which contains information on 2280 drug metabolites [38]; and (3) PubChem, which contains live record counts of 94,017,529 compounds (mostly small molecules) with data on chemical structures, identifiers, chemical, and physical properties, biological activities, patents, health, safety, toxicity data, and other properties [39]. In addition, search engines including Google Scholar and SciFinder Scholar were used for tracing the references on biological activities of each compound. Unique search terms/keywords including "biological activities", "anti-inflammatory", and "immune regulation" were used. Content analyses of these databases and 
references were performed focusing on the reported biological activities, and this information was then tabulated and cited against each compound.

\section{Conclusions}

We have shown that $D$. caninum excrete/secrete as many as 35 known polar metabolites and 14 fatty acids, and that GC-MS and LC-MS can be used to profile the metabolite complement of tapeworm ESP. While most of the polar metabolites belong to the chemotaxonomy of organic acids (with nine small molecules), most of the non-polar metabolites were saturated fatty acids. Four unsaturated fatty acids and one SCFA were also detected in the non-polar faction of D. caninum ESP. PCA analysis showed clear separation of $D$. caninum secreted metabolites from culture media components, and the major metabolite was identified as succinic acid. Of a total of 49 metabolites identified, 12 have known pharmacological properties, notably as anti-inflammatory agents. These bioactive molecules may be, individually or in combination, responsible for the immune dampening effects of tapeworms in their definitive mammalian hosts, allowing them to survive in such hostile environments as the gut. Future work will entail purification and isolation of pharmacologically bioactive compounds from D. caninum ESP and assessment of their anti-inflammatory properties at physiologically relevant concentrations in appropriate in vitro and in vivo settings.

Author Contributions: Conceptualization, methodology, investigation, data curation, software analyses, validation, writing-original draft preparation by P.W.; worm collection by C.C.; draft corrections by M.F. and R.M.E.; and resources, supervision, project administration, funding acquisition and writing-review and editing by A.L.

Funding: This research was funded by a NHMRC Peter Doherty Early Career Researcher fellowship (APP1091011) and AITHM Capacity Development Grant to P.W.; NHMRC program grant (APP1037304), and senior principal research fellowship (APP1117504) to A.L.

Acknowledgments: This work was made possible due to the GC-MS and LC-MS support provided by Malcolm McConville and Konstantinos Kouremenos of Bio 21 Institute, University of Melbourne, Australia.

Conflicts of Interest: The authors declare no conflict of interest. The funders had no role in the design of the study; in the collection, analyses, or interpretation of data; in the writing of the manuscript, or in the decision to publish the results.

\section{References}

1. WHO. Taeniasis/Cysticercosis: Fact Sheet. World Health Organisation, Avenue Appia 20, 1202 Geneva. 2019. Available online: https:/www.who.int/news-room/fact-sheets/detail/taeniasis-cysticercosis (accessed on 20 March 2019).

2. CDC. Dipylidium Caninum; Centre for Disease Control and Prevention, Global Health, Division of Parasitic Diseases and Malaria, 2017. Available online: https:/www.cdc.gov/dpdx/dipylidium/index.html (accessed on 20 March 2019).

3. Coakley, G.; Buck, A.H.; Maizels, R.M. Host parasite communications-Messages from helminths for the immune system parasite communication and cell-cell interactions. Mol. Biochem. Parasitol. 2016, 208, 33-40. [CrossRef]

4. Pan, W.; Hao, W.T.; Shen, Y.J.; Li, X.Y.; Wang, F.F.S.; Sun, F.-F.; Yin, J.H.; Zhang, J.; Tang, R.X.; Cao, J.-P.; et al. The excretory-secretory products of Echinococcus granulosus protoscoleces directly regulate the differentiation of B10, B17 and Th17 cells. Parasit. Vectors 2017, 10, 348. [CrossRef] [PubMed]

5. Nono, J.K.; Pletinckx, K.; Lutz, M.B.; Brehm, K. Excretory/secretory-products of Echinococcus multilocularis larvae induce apoptosis and tolerogenic properties in dendritic cells in vitro. PLoS Negl. Trop. Dis. 2012, 6, e1516. [CrossRef] [PubMed]

6. $\quad$ Reyes, J.L.; Lopes, F.; Leung, G.; Jayme, T.S.; Matisz, C.E.; Burkhard, R.; Carneiro, M.; Workentine, M.L.; Wang, A.; Petri, B.; et al. Macrophages treated with antigen from the tapeworm Hymenolepis diminuta condition CD25+ T cells to suppress colitis. FASEB J. 2019, 33, 5676-5689. [CrossRef] [PubMed] 
7. Graves, N.; Venu, V.P.; Yipp, B.G.; Petri, B.; Hirota, S.; Gilleard, J.; McKay, D.M.; Lopes, F. A trypsin-sensitive proteoglycan from the tapeworm Hymenolepis diminuta inhibits murine neutrophil chemotaxis in vitro by suppressing p38 MAP kinase activation. J. Innate Immun. 2019, 11, 136-149. [CrossRef] [PubMed]

8. Reyes, J.L.; Lopes, F.; Leung, G.; Mancini, N.L.; Matisz, C.E.; Wang, A.; Thomson, E.A.; Graves, N.; Gilleard, J.; McKay, D.M. Treatment with cestode parasite antigens esults in recruitment of CCR2+ myeloid cells, the adoptive transfer of which ameliorates colitis. Infect. Immun. 2016, 84, 3471-3483. [CrossRef] [PubMed]

9. Lima, E.O.; Esteves, C.Z.; Oliveira, D.N.; Guerreiro, T.M.; Melo, C.F.O.R.; Catharino, R.R. Mass Spectrometry and Metabolomics-New Approaches for Helminth Biochemical Studies in Human Helminthiasis; Rodrigo, L., Ed.; InTechOpen Limited: London, UK, 2017.

10. Shepherd, C.; Navarro, S.; Wangchuk, P.; Wilson, D.; Daly, N.L.; Loukas, A. Identifying the immunomodulatory components of helminths. Parasit Immunol. 2015, 37, 293-303. [CrossRef] [PubMed]

11. Carlson, D.A.; Langley, P.A.; Huyton, P. Sex pheromone of the tsetse fly: isolation, identification, and synthesis of contact aphrodisiacs. Science 1978, 201, 750-753. [CrossRef]

12. Doonan, J.; Lumb, F.E.; Pineda, M.K.; Tarafdar, A.; Crowe, J.; Khan, A.M.; Suckling, C.J.; Harnett, M.M.; Harnett1, W. Protection against arthritis by the parasitic worm product ES-62, and its drug-like small molecule analogues, is associated with inhibition of osteoclastogenesis. Front. Immunol. 2018, 9. [CrossRef]

13. Wangchuk, P.; Shepherd, C.; Constantinoiu, C.; Ryan, R.Y.M.; Kouremenos, K.A.; Becker, L.; Buitrago, G.; Giacomin, P.; Wilson, D.; Daly, N.; et al. Hookworm-derived metabolites suppress pathology in a mouse model of colitis and inhibit secretion of key inflammatory cytokines in primary human leukocytes. Infect. Immun. 2019. [CrossRef]

14. Hearps, A.C.; Tyssen, D.; Srbinovski, D.; Bayigga, L.; Diaz, D.J.D.; Aldunate, M.; Cone, R.A.; Gugasyan, R.; Anderson, D.J.; Tachedjian, G. Vaginal lactic acid elicits an anti-inflammatory response from human cervicovaginal epithelial cells and inhibits production of pro-inflammatory mediators associated with HIV acquisition. Mucosal. Immunol. 2017, 10, 1480-1490. [CrossRef]

15. Tang, X.; Liu, J.; Dong, W.; Li, P.; Li, L.; Lin, C.; Zheng, Y.; Hou, J.; Li, D. The cardioprotective effects of citric Acid and L-malic acid on myocardial ischemia/reperfusion injury. Evid. Based Complement. Altern. Med. 2013, 2013, 820695. [CrossRef]

16. Unnikrishnan, M.K.; Rao, M.N. Antiinflammatory activity of methionine, methionine sulfoxide and methionine sulfone. Agents Actions 1990, 31, 110-112. [CrossRef] [PubMed]

17. Han, D.; Kim, H.Y.; Lee, H.J.; Shim, I.; Hahm, D.H. Wound healing activity of gamma-aminobutyric Acid (GABA) in rats. J. Microbiol. Biotechnol. 2007, 17, 1661-1669.

18. Szél, E.; Polyánka, H.; Szabó, K.; Hartmann, P.; Degovics, D.; Balázs, B.; Nemeth, I.B.; Korponyai, C.; Csanyi, E.; Kaszaki, J.; et al. Anti-irritant and anti-inflammatory effects of glycerol and xylitol in sodium lauryl sulphate-induced acute irritation. J. Eur. Acad. Dermatol. Venereol. 2015, 29, 2333-2341. [CrossRef] [PubMed]

19. Glushakova, O.; Kosugi, T.; Roncal, C.; Mu, W.; Heinig, M.; Cirillo, P.; Sanchez-Lozada, L.G.; Johnson, R.J.; Nakagawa, T. Fructose induces the inflammatory molecule ICAM-1 in endothelial cells. J. Am. Soc. Nephrol. 2008, 19, 1712-1720. [CrossRef]

20. Ahmad, M.; Baba, W.N.; Gani, A.; Wani, T.A.; Gani, A.; Masoodi, F.A. Effect of extraction time on antioxidants and bioactive volatile components of green tea (Camellia sinensis), using GC/MS. Cogent Food Sci. Agric. 2015, 1, 1106387. [CrossRef]

21. Aparna, V.; Dileep, K.V.; Mandal, P.K.; Karthe, P.; Sadasivan, C.; Haridas, M. Anti-inflammatory property of n-hexadecanoic acid: structural evidence and kinetic assessment. Chem. Biol. Drug Des. 2012, 80, 434-439. [CrossRef]

22. Kuda, O. Bioactive metabolites of docosahexaenoic acid. Biochimie 2017, 136, 12-20. [CrossRef]

23. Tedelind, S.; Westverg, F.; Kjerrulf, M.; Vidal, A. Anti-inflammatory properties of the short-chain fatty acids acetate and propionate: A study with relevance to inflammatory bowel disease. World J. Gastroenterol. 2007, 13, 2826-2832. [CrossRef] [PubMed]

24. Huang, W.C.; Tsai, T.H.; Chuang, L.T.; Li, Y.Y.; Zouboulis, C.C.; Tsai, P.J. Antibacterial and anti-inflammatory properties of capric acid against propionibacterium acnes: A comparative study with lauric acid. J. Dermatol. Sci. 2014, 73, 232-240. [CrossRef]

25. Wang, Y.; Li, J.V.; Saric, J.; Keiser, J.; Wu, J.; Utzinger, J.; Holmes, E. Advances in metabolic profiling of experimental nematode and trematode infections. Adv. Parasitol. 2010, 73, 373-404. 
26. Beames, C.G.J. Neutral lipids of Ascaris lumbricoides with special reference to the esterified fatty acids. Exp. Parasitol. 1965, 16, 291-299. [CrossRef]

27. Kaplan, F.; Srinivasan, J.; Mahanti, P.; Ajredini, R.; Durak, O.; Nimalendran, R.; Sternberg, P.W.; Teal, P.E.; Schroeder, F.C.; Edison, A.S.; et al. Ascaroside expression in Caenorhabditis elegans is strongly dependent on diet and developmental stage. PLoS ONE 2011, 6, e17804. [CrossRef]

28. Wishart, D.S.; Jewison, T.; Guo, A.C.; Wilson, M.; Knox, C.; Liu, Y.; Djoumbou, Y.; Mandal, R.; Aziat, F.; Dong, E.; et al. HMDB 3.0—The human metabolome database in 2013. Nucleic Acids Res. 2013, 41, D801-D807. [CrossRef]

29. Belzer, C.; Chia, L.W.; Aalvink, S.; Chamlaqain, B.; Piironen, V.; Knol, J.; de Vos, W.M. Microbial metabolic networks at the mucus layer lead to diet-independent butyrate and vitamin B12 production by intestinal symbionts. MBio 2017, 8, e00770-17. [CrossRef]

30. Loukas, A.; Hotez, P.J.; Diemert, D.; Yazdanbakhsh, M.; McCarthy, J.S.; Correa-Oliveira, R.; Croese, J.; Bethony, J.M. Hookworm infection. Nat. Rev. Dis. Primers. 2016, 2, 16088. [CrossRef]

31. Maizels, R.M. Parasitic helminth infections and the control of human allergic and autoimmune disorders. Clin. Microbiol. Infect. 2016, 22, 481-486. [CrossRef]

32. Maizels, R.M.; Smits, H.H.; McSorley, H.J. Modulation of host immunity by helminths: The expanding repertoire of parasite effector molecules. Immunity 2018, 49, 801-818. [CrossRef]

33. Scheppach, W. Effects of short chain fatty acids on gut morphology and function. Gut 1994, 35, S35-S38. [CrossRef]

34. Gill, P.A.; van Zelm, M.C.; Muir, J.G.; Gibson, P.R. Review article: Short chain fatty acids as potential therapeutic agents in human gastrointestinal and inflammatory disorders. Aliment. Pharmacol. Ther. 2018, 48, 15-34. [CrossRef]

35. Overgaard, A.J.; Weir, J.M.; De Souza, D.P.; Tull, D.; Haase, C.; Meikle, P.J.; Pociot, F. Lipidomic and metabolomic characterization of a genetically modified mouse model of the early stages of human type 1 diabetes pathogenesis. Metabolomics 2016, 12, 13. [CrossRef] [PubMed]

36. Han, J.; Lin, K.; Sequeia, C.; Borchers, C.H. An isotope-labeled chemical derivatization method for the quantitation of short-chain fatty acids in human feces by liquid chromatography-tandem mass spectrometry. Anal. Chim. Acta 2015, 854, 86-94. [CrossRef] [PubMed]

37. Xia, J.; Psychogios, N.; Young, N.; Wishart, D.S. MetaboAnalyst: A web server for metabolomic data analysis and interpretation. Nucleic Acids Res. 2009, 37, W652-W660. [CrossRef]

38. Wishart, D.S.; Feunang, Y.D.; Guo, A.C.; Lo, E.J.; Marcu, A.; Grant, J.R.; Sajed, T.; Johnson, D.; Li, C.; Sayeeda, Z.; et al. DrugBank 5.0: A major update to the DrugBank database for 2018. Nucleic Acids Res. 2017, 46, D1074-D1082. [CrossRef]

39. Kim, S.; Thiessen, P.A.; Bolton, E.E.; Chen, J.; Fu, G.; Gindulyte, A.; Han, L.; He, J.; He, S.; Shoemaker, B.A.; et al. PubChem substance and compound databases. Nucleic Acids Res. 2016, 44, D1202-D1213. [CrossRef] [PubMed]

Sample Availability: Excretory-secretory products of tapeworms/samples are available from the authors at Australian Institute of Tropical Health and Medicine, James Cook University, Cairns, Australia.

(C) 2019 by the authors. Licensee MDPI, Basel, Switzerland. This article is an open access article distributed under the terms and conditions of the Creative Commons Attribution (CC BY) license (http://creativecommons.org/licenses/by/4.0/). 